Karavin, M, Çalışkan Dede, E, Dede, O. International Journal of Science Letters (IJSL). 2021. 3(1):

32-51.

https://doi.org/10.38058/ijsl.853982

Research Article

\title{
Determination of Auchenorrhyncha species distributed in apple orchards in Amasya, Turkey with a new record for Turkish fauna
}

\author{
Murat Karavin $^{1 *}$ DD , Elif Çalışkan Dede ${ }^{1}$ ID, Onur Dede $^{1}$ \\ ${ }^{1}$ Department of Plant and Animal Production, Suluova Vocational School, Amasya \\ University, Amasya/Turkey
}

\begin{abstract}
Apple is the economically most important agricultural product in

Amasya. So, identifiying the potentially pest species in apple orchards

is very important for agriculture and economy. Auchenorrhyncha

species, known as plant pests, adversely affect the growth of plants directly or indirectly and as a result cause abnormal development, damage and even death of plant tissues and cells. In this study, it was aimed to determine the Auchenorrhyncha species which are potentially pests in apple orchards in Amasya, Turkey. 38 species belonging to the Auchenorrhyncha were determined in apple orchards in Amasya and Trypetimorpha occidentalis is recorded for the first time for the Turkish Auchenorrhyncha fauna.
\end{abstract}

Article History

Received 15.10.2020

Accepted 21.12.2020

\author{
Keywords \\ Hemiptera, \\ Auchenorrhyncha, \\ Apple, \\ Fauna, \\ IPM
}

\section{Introduction}

Auchenorrhyncha is one of the sub-order belonging to the order Hemiptera (Insecta), which has great number of families. The functional significance of the insects belonging to this group is little known. Since they are in high numbers in tree, shrub and herb layers, the biomass of them are high in vegetation. Because of this, they are significant components of terrestrial food chain. Insects belonging to this group move by jumping. There are mottling and patterning in different colors and shapes on the body and wings. At the rest position, the wings rest on the abdomen to form a roof.

By means of their piercing-absorbent mouth structure, they feed by sucking plant sap from the intracellular, intercellular and transmission bundles of the plants. Therefore, species belonging to this group known as plant pests and affect the development of plants directly or

\footnotetext{
${ }^{1}$ Correspondence: murat.karavin@amasya.edu.tr
} 
indirectly. In addition, they secrete toxic substances while sucking sap from the plant, which cause abnormal development, damage, or even death of plant tissues and cells. The species belonging to the Auchenorrhyncha group are damage plants directly by feeding and laying eggs, and indirectly by blocking the conduction tissues during feeding and carrying and spreading viral and mycoplasmal diseases (Ossiannilsson, 1978). The diagnosis of Auchenorrhyncha species is usually based on morphological characters. Some of these characters are visible from the outside, and some are visible as a result of dissection (Holzinger et al., 2003). In the part after the seventh abdominal segment removed from the body, genital structures bearing important taxonomically reliable characters are aedeagus, stylus, pygofer, genital plate, pregenital sternitis in men, and the seventh pregenital sternitis and ovipositor in female. In order to clash against Auchenorrhyncha species, which are very important in economic terms, it is necessary to know the species existing in nature, the density of their population and their distributions. The detection of these species is also important in terms of Turkey's biodiversity and to demonstrate Auchenorrhyncha fauna.

The first study on Auchenorrhyncha suborder in Turkey was carried out by Fahringer (1922). Fahringer (1922) listed some species from Turkey and then Dlabola (1957, 1971a, 1971b, 1974, 1979, 1985, 1987), Lodos and Kalkandelen (1985a, 1985b, 1985c, 1986a, 1986b, 1986c, 1987a, 1987b, 1987c, 1987d, 1988), Kartal and Zeybekoğlu (1991, 1992, 1994a, 1994b, 1996, 1997) studied on Auchenorrhyncha fauna. But, there are no studies on Auchenorrhyncha species in economically important apple orchards.

In the literature, only one study was found on Auchenorrhyncha species in apple orchards in Turkey. Ayaz and Yücel (2010) reported five species belonging to Auchenorrhyncha in apple orchards in Elazığ. In addition, Bleicher, Markó and Orosz (2006) determined 109 species in two apple orchards in Hungary. Bleicher, Orosz, Cross and Markó (2010) determined 77 species in three apple orchards in England.

In this study, it was aimed to determine the Auchenorrhyncha species which are potentially pests in apple orchards in Amasya, Turkey. Apple is the economically most important agricultural product in Amasya. So, identifiying the potentially pest species in apple orchards is very important for agriculture and economy. 


\section{Materials and Methods}

The Auchenorrhyncha species used as research materials were collected from the apple orchards selected from different localities in Amasya. Samples were collected with sweep net by hitting the trunk, branch and leaf parts of the apple trees and weeds in the orchards. Insect samples were prepared according to standart methods. In Auchenorrhyncha specimens, species diagnosis is made according to the shape and structural features of the genital organs. In the male and female insect, the end part after the seventh segment of the abdomen where the genital organs are located, was detached from the body with the help of a dissection needle in a stereo microscope. The shapes and structures of these genital parts are taxonomically reliable characters for identifiying. Each sample was examined in detail in terms of body shape, structure, genital structures, color, patterning characters under the stereomicroscope, and identified compared with the definitions given in the literature for the relevant taxa and the previously diagnosed samples.

\section{Results and Discussion}

As a result of the research, 38 species belonging to 8 families of the suborder Auchenorrhyncha were detemined in apple orchards of Amasya Province. These species are;

\section{Cixiidae}

Pentastira rorida (Fieber, 1876)

Material examined: $1 q, 28.07 .2016$, Y1k1lgan Village, Amasya

Distrubioun in Turkey: Balıkesir, Edirne, Erzincan, İzmir, Manisa, Mardin, Niğde, Tokat (Önder et al., 2011).

Zoogeographical distribution: Southeast of Europe, Eastern Mediterranean and Southeast of Central Europe (Holzinger et al., 2003)

\section{Reptalus cuspidatus (Fieber, 1876)}

Material examined: $1 \delta^{\Uparrow} 1$, 18.07.2016, Merzifon; 1 , 08.06.2016, Ayrancı Village, Suluova; 28.06.2016, Ziyaret, Amasya.

Zoogeographical distribution: Europe and Central Asia (Holzinger et al., 2003) 


\section{Delphacidae}

Asiraca clavicornis (Fabricius, 1794)

Material examined: $1 \hat{\jmath}, 06.08 .2016$, Yenimahalle, Suluova; $1 \hat{\delta}, 07.09 .2106$, Korkut, Hamamözü, Amasya.

Distrubution in Turkey: Ankara, Antalya, Aydın, Çorum, Denizli, Erzurum, İzmir, İstanbul, Konya, Kütahya, Muğla, Samsun, Sinop, Tokat, Yozgat (Lodos and Kalkandelen, 1980; Asche, 1982; Güçlü, 1996; Karavin, 2012).

Zoogeographical Distribution: Afghanistan, Albania, Armenia, Austria, Azerbaijan, Belgium, Bulgaria, China, Cyprus, Czech Republic, England, France, Georgia, Germany, Hungary, Ireland, Israel, Italy, Kazakhstan, Kyrgyzstan, Latvia, Moldavia, Morocco, Netherlands, Poland, Portugal, Romania, Slovakia, Spain, Switzerland, Tajikistan, Tunisia, Turkmenistan, Ukraine, Uzbekistan, Yugoslavia (Nast, 1972).

Kelisia sabulicola Wagner, 1952

Material examined: $1 \lesssim, 06.08 .2016$, Yenimahalle, Suluova, Amasya.

Distrubution in Turkey: Amasya, Çorum, Samsun, Sinop, Tokat (Karavin, 2012).

Zoogeographical Distribution: Austria, France, Germany, Greece, Hungary, Italy (Holzinger et al., 2003).

\section{Javesella dubia (Kirschbaum, 1868)}

Material examined: $1 \widehat{\jmath}, 06.08 .2016$, Ayranc1 Village, Suluova, Amasya.

Distrubution in Turkey: Amasya, Ankara, Çorum, Erzurum, Ordu, Samsun, Tokat (Lodos and Kalkandelen, 1980; Asche, 1982; Güçlü, 1996; Karavin, 2012).

Zoogeographical Distribution: Austria, Azores Islands, Belgium, Bulgaria, Cyprus, Czech Republic, Denmark, England, Estonia, Finland, France, Germany, Ireland, Italy, Kazakhstan, Latvia, Moldavia, Morocco, Netherlands, Norway, Poland, Romania, Slovakia, Sweden, UkraineUzbekistan (Nast, 1972). 


\section{Laodelphax striatellus (Fallén, 1826)}

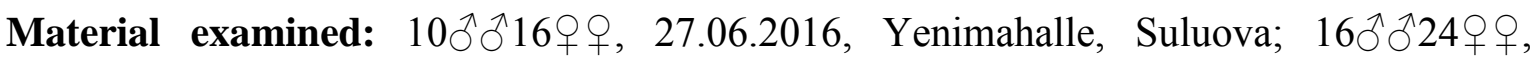

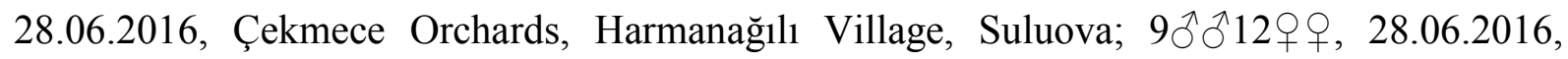

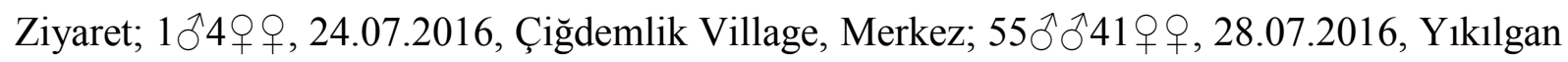

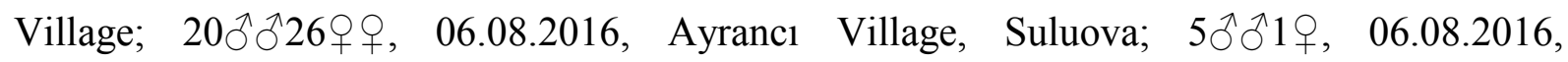
Yenimahalle, Suluova; $3 \widehat{\delta} 2 \rho+$, 10.08.2016, Dutlupınar, Gümüşhacıköy; $7 \delta^{\Uparrow} 2 q \circ$,

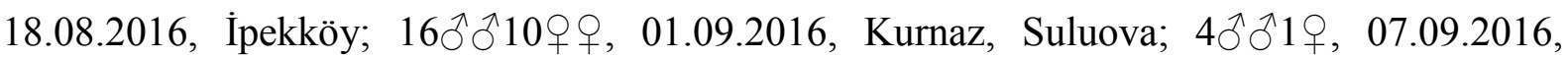
Alıcık-Merzifon, Amasya.

Distrubution in Turkey: Adana, Adıyaman, Amasya, Ankara, Antalya, Bilecik, Bitlis, Çorum, Diyarbakır, Elazığg, Erzincan, Erzurum, Eskişehir, Iğdır, İçel, İzmir, Kahramanmaraş, Kars, Malatya, Mersin, Muğla, Niğde, Ordu, Rize, Samsun, Siirt, Sinop, Şırnak, Tokat (Dlabola, 1957; Lodos and Kalkandelen, 1980; Asche, 1982; Güçlü, 1996; Karavin, 2012; Karavin and Özgen, 2017; Gözüaçık and Özgen, 2018).

Zoogeographical Distribution: Afghanistan, Albania, Algeria, Armenia, Austria, Azerbaijan, Bulgaria, Canary Islands, China, Czech Republic, England, Estonia, Finland, France, Georgia, Germany, Hungary, Iran, Iraq, Israel, Italy, Japan, Kazakhstan, Korea, Kyrgyzstan, Latvia, Lebanon, Moldavia, Mongolia, Netherlands, Poland, Portugal, Romania, Russia, Slovakia, Spain, Sweden, Tajikistan, Tunisia, Turkey, Ukraine, Uzbekistan, Yugoslavia (Nast, 1972).

\section{Ribautodelphax albostriata (Fieber, 1866)}

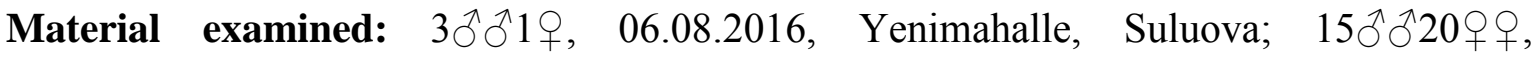
07.09.2016, Korkut, Hamamözü, Amasya.

Distrubution in Turkey: Ankara, Erzincan, Tokat (Lodos and Kalkandelen, 1988; Karavin, 2012).

Zoogeographical Distribution: Austria, Belgium, Caucasus, Cyprus, Czech Republic, Denmark, Estonia, Finland, France, Germany, Hungary, Italy, Kazakhstan, Kyrgyzstan, Moldavia, Mongolia, Netherlands, Norway, Poland, Portugal, Romania, Russia, Siberia, Slovakia, Spain, Sweden, Switzerland, Tunisia, Ukraine, Yugoslavia (Nast, 1972). 


\section{Toya propinqua (Fieber, 1866)}

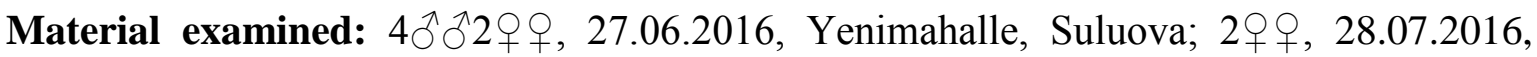

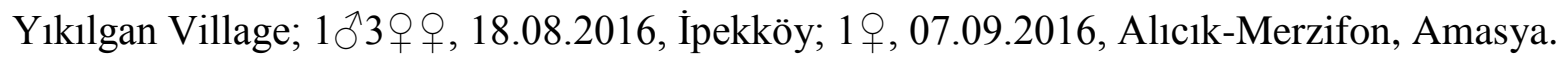

Distrubution in Turkey: Adana, Afyon, Amasya, Ankara, Antalya, Aydın, Çanakkale, Çorum, Denizli, Diyarbakır, Erzurum, Gaziantep, Hatay, Isparta, İçel, İskenderun, Kastamonu, Mardin, Muğla, Ordu, Samsun, Siirt, Sinop, Şırnak (Silopi),Tokat (Dlabola, 1957; Lodos and Kalkandelen, 1980; Asche, 1982; Güçlü, 1996; Karavin, 2012; Karavin and Özgen, 2017).

Zoogeographical Distribution: Afghanistan, Albania, Algeria, Armenia, Austria, Bulgaria, Canary Islands, Cyprus, Czech Republic, Egypt, France, Georgia, Germany, Hungary, Iran, Iraq, Israel, Italy, Japan, Jordan, Kazakhstan, Kyrgyzstan, Libya, Moldavia, Morocco, Poland, Portugal, Romania, Russia, Slovakia, Spain, Tajikistan, Tunisia, Turkey, Uzbekistan, Yugoslavia (Nast, 1972).

\section{Dictyopharidae}

Dictyophara europaea (Linné, 1767)

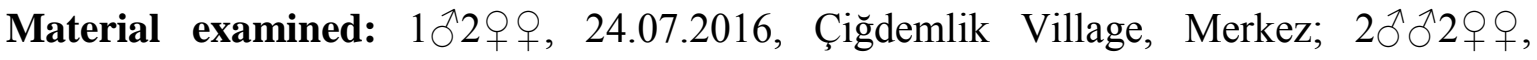
06.08.2016, Ayranc1 Village, Suluova; $2 \lesssim ð 2 q \circ, 06.08 .2016$, Yenimahalle, Suluova, Amasya.

Distrubution in Turkey: Ankara, Denizli, Diyarbakır, Elazığ, Eskişehir, Isparta, İstanbul, İzmir, Kars, Kastamonu, Manisa, Mardin, Muğla, Muş, Ordu, Samsun, Siirt, Van (Özgen et al., 2009; Önder et al., 2011).

Zoogeographical Distribution: Afghanistan, Albania, Algeria, Armenia, Austria, Azerbaijan, Belgium, Bulgaria, China, Czech Republic, France, Georgia, Germany, Greece, Hungary, Iraq, Italy, Kazakhstan, Kyrgyzstan, Moldavia, Poland, Portugal, Romania, Russia, Slovakia, Spain, Switzerland, Tunisia, Ukraine, Uzbekistan, Yugoslavia (Nast, 1972).

\section{Tropiduchidae}

Trypetimorpha occidentalis Huang \& Bourgoin, 1993

Material examined: $1 \delta, 28.06 .2016$, Çekmece Orchards, Harmanağılı Village, Suluova, Amasya. 
Distrubution in Turkey: New record for the Turkish Auchenorrhyncha Fauna.

Zoogeographical Distribution: Armenia, Austria, Bulgaria, Cyprus, Czech Republic, France, Hungary, Israel, Italy, Kyrgyzstan, Moldavia, Romania, Russia, Slovakia, Ukraine (Nast, 1972).

\section{Issidae}

Agalmatium flavescens (Olivier, 1791)

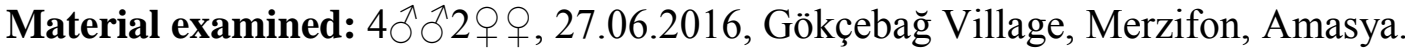

Distribution in Turkey: Ankara, Bursa, Çorum, İstanbul, Edirne, Kastamonu, Tekirdağ, Van, Zonguldak (Önder et al., 2011).

Zoogeographical Distribution: Albania, Algeria, Austria, Bulgaria, Cyprus, Czech Republic, France, Greece, Hungary, Italy, Jordan, Morocco, Portugal, Romania, Slovakia, Spain, Switzerland, the Canary Islands, Turkey (Nast, 1972; Önder et al., 2011).

\section{Agalmatium bilobum (Fieber, 1877)}

Material examined: $1 \delta, 27.06 .2016$, Yenimahalle, Suluova, Amasya.

Distribution in Turkey: Adıyaman, Afyonkarahisar, Amasya, Ankara, Aydın, Balıkesir, Bilecik, Burdur, Bursa, Çanakkale, Çorum, Denizli, Eskişehir, Gaziantep, Gümüşhane, İzmir, Kütahya, Malatya, Manisa, Muğla, Tekirdağ, Tokat, Uşak (Önder et al., 2011).

Zoogeographical Distribution: France, Greece, Israel, Italy, Russia, Spain, Syria, Tunisia, Turkey (Lodos and Kalkandelen, 1981).

\section{Aphrophoridae \\ Aphrophora alni (Fallen, 1805)}

Material examined: $1 \hat{\jmath}, 07.09 .2016$, Korkut, Hamamözü, Amasya.

Distribution in Turkey: Adana, Afyonkarahisar, Ankara, Artvin, Aydın, Balıkesir, Bitlis, Bolu, Çanakkale, Çorum, Diyarbakır, Erzincan, Erzurum, Giresun, İstanbul, İzmir, Kayseri, Kırklareli, Konya, Kütahya, Mardin, Manisa, Muğla, Ordu, Rize, Samsun, Sinop, Tekirdağ, Trabzon, Yozgat (Önder et al., 2011).

Zoogeographical Distribution: Albania, Algeria, Armenia, Austria, Azerbaijan, Belgium, Bulgaria, China, Czech Republic, Denmark, England, Estonia, Finland, Georgia, Germany, 
Greece, Hungary, Ireland, Italy, Japan, Kazakhstan, Moldova, Mongolia, Morocco, Netherlands, Norway, Poland, Portugal, Romania, Russia, Slovakia, Spain, Sweden, Switzerland, Turkey, Turkmenistan, Ukraine (Nast, 1972; Önder et al., 2011).

\section{Lepyronia coleoptrata (Linné, 1758)}

Material examined: $1 \widehat{\partial}, 27.06 .2016$, Yenimahalle, Suluova; $1 \hat{\jmath}, 18.07 .2016$, Merzifon; $1 \precsim 1$, 10.08.2016, Dutlupınar, Gümüşhacıköy, Amasya.

Distribution in Turkey: Adana, Afyonkarahisar, Ankara, Artvin, Aydın, Bilecik, Bursa, Çanakkale, Çankırı, Çorum, Diyarbakır, Edirne, Elazı̆̆g, Erzincan, Gümüşhane, İzmir, Kahramanmaraş, Kars, Kütahya, Manisa, Mardin, Muğla, Muş, Sakarya, Siirt, Tokat (Önder et al., 2011; Özgen et al., 2018).

Zoogeographical Distribution: Afghanistan, Albania, Algeria, Austria, Belgium, Bulgaria, Czech Republic, Denmark, Finland, France, Germany, Greece, Hungary, Iraq, Italy, Mongolia, Netherlands, Norway, Poland, Portugal, Romania, Russia, Slovakia, Spain, Sweden, Switzerland, Syria, Turkey (Önder et al., 2011).

\section{Philaenus spumarius (Linne, 1758)}

Material examined: $1 \delta^{\Uparrow} 1$ q, 27.06.2016, Gökçebağ Village, Merzifon; 1 $\widehat{\jmath}, 28.06 .2016$, Ziyaret, Amasya.

Distribution in Turkey: Ağrı, Amasya, Ankara, Artvin, Aydın, Balıkesir, Bilecik, Bitlis, Bolu, Bursa, Çanakkale, Elazığg, Erzincan, Erzurum, Eskişehir, Giresun, Gümüşhane, Hakkari, İstanbul, İzmir, Kars, Kırklareli, Kütahya, Kocaeli, Malatya, Manisa, Mardin, Muğla, Ordu, Rize, Samsun, Siirt, Sinop, Tekirdağ, Trabzon, Tokat, Van (Önder et al., 2011; Özgen et al., 2018).

Zoogeographical Distribution: Afghanistan, Albania, Algeria, Austria, Azores, Balearic Islands, Belgium, Bulgaria, China, Cyprus, Czech Republic, Denmark, England, Finland, France, Germany, Greece, Hungary, Iraq, Ireland, Italy, Japan, Mongolia, Morocco, Netherlands, Norway, Poland, Portugal, Romania, Russia, Slovakia, Sweden, Switzerland, Syria, Tunisia, Turkey (Önder et al., 2011). 


\section{Membracidae \\ Stictocephala bisonia Kopp \& Yonke, 1977}

Material examined: $1 \delta, 28.06 .2016$, Ziyaret; $1 \widehat{\jmath}, 24.07 .2016$, Çiğdemlik Village; $1 \hat{\jmath} 19$, 28.07.2016, Y1k1lgan Village; $1 \delta^{\Uparrow} 1$, 06.08.2016, Yenimahalle, Suluova; 1q, 18.08.2016, İpekköy, Amasya.

Zoogeographical Distribution: North America, Europe, Central Asia (Holzinger et al., 2003).

\section{Cicadellidae}

Anaceratagallia laevis (Ribaut, 1935)

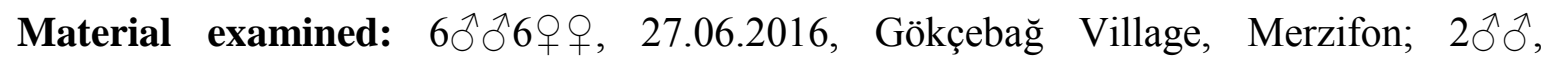
27.06.2017, Yenimahalle, Suluova; 1ðึ, 28.06.2016, Çekmece Orchards, Harmanağılı Village,

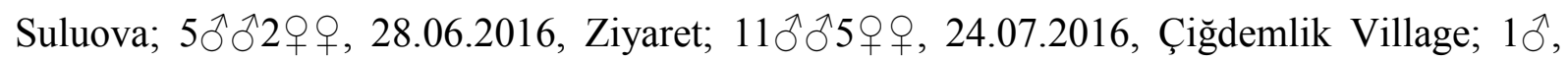

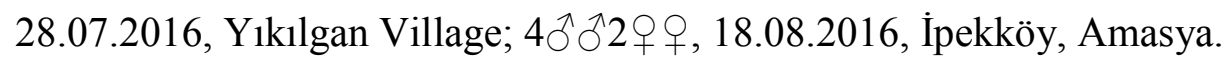

Distribution in Turkey: Adana, Ağrı, Ankara, Bilecik, Erzurum, Eskişehir, İçel, İstanbul, İzmir, Konya, Malatya, Nevşehir (Önder et al., 2011).

Zoogeographical Distribution: Afghanistan, Albania, Bulgaria, Cyprus, Egypt, France, Greece, Hungary, Iran, Iraq, Israel, Italy, Jordan, Morocco, Portugal, Romania, Russia, the Canary Islands, Turkey (Nast, 1972; Önder et al., 2011).

\section{Anaceratagallia ribauti (Ossiannilsson, 1938)}

Material examined: $3 \widehat{\partial}, 27.06 .2016$, Yenimahalle, Suluova; 4$\widehat{\partial}, 06.08 .2016$, Ayranc1 Village, Suluova; $4 \bigcirc^{\Uparrow} 2$ + $ᄋ$, 10.08.2016, Merzifon; $1 \overbrace{}^{\Uparrow} 1+$, 07.09.2016, Korkut, Hamamözü, Amasya.

Distribution in Turkey: Adana, Ankara, Balıkesir, Çankırı, Elazığ, Hatay, İçel, İzmir, Malatya, Mardin, Samsun (Önder et al., 2011; Kaplan and Yücel, 2014).

Zoogeographical Distribution: England, Europe, Iran, Russia, Turkey (Önder et al., 2011). 
Material examined: 1q, 27.06.2016, Yenimahalle, Suluova; $3 \hat{\jmath} 2 q \circ$, 18.07.2016, Merzifon, Amasya.

Distribution in Turkey: Adana, Adıyaman, Afyonkarahisar, Ankara, Artvin, Çanakkale, Diyarbakır, İçel, Kahramanmaraş, Konya, Malatya, Mardin, Muğla, Nevşehir, Niğde, Şanlıurfa (Önder et al., 2011; Tolga and Yoldaş, 2019).

Zoogeographical Distribution: Algeria, Canary Islands, Cyprus, Europe, Iran, Iraq, Ireland, Israel, Mongolia, Morocco, Russia, Syria, Tunisia, Turkey (Nast, 1972; Önder et al., 2011).

\section{Aphrodes albifrons (Linnaeus 1758)}

Material examined: $1 \delta, 27.06 .2016$, Gökçebağ Village, Merzifon, Amasya.

Distribution in Turkey: İzmir, Manisa (Önder et al., 2011).

Zoogeographical Distribution: Israel, Spain, Turkey (Önder et al., 2011).

\section{Aphrodes bicinctus (Zachvatkin 1948)}

Material examined: $19,27.06 .2016$, Yenimahalle, Suluova, Amasya.

Distribution in Turkey: All over the Turkey (Önder et al., 2011).

Zoogeographical Distribution: Afghanistan, Algeria, Britain, Cyprus, Europe, Iran, Ireland, Lebanon, Madeira Island, Mongolia, Morocco, North America, Russia, Syria, Tunisia, Turkey (Nast, 1972; Önder et al., 2011).

\section{Cicadella viridis (Linnaeus, 1758)}

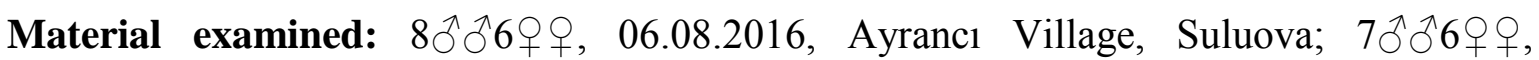
18.08.2016, İpekköy, Amasya.

Distribution in Turkey: Amasya, Artvin, Balıkesir, Bursa, Çanakkale, Diyarbakır, Edirne, Erzincan, Erzurum, İzmir, Kars, Kırklareli, Manisa, Mardin, Muğla, Samsun, Tekirdağ (Önder et al., 2011).

Zoogeographical Distribution: China, Europe, Iran, Ireland, Japan, Korea, Mongolia, Russia, Turkey (Önder et al., 2011). 


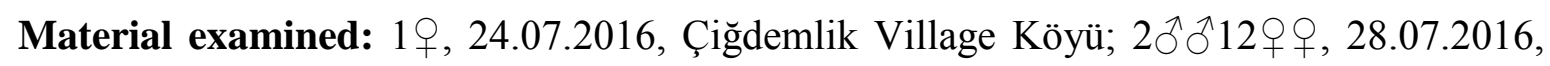
Yık1lgan Village; $3 \widehat{\jmath} 11$ 우, 10.08.2016, Dutlupınar, Gümüşhacıköy, Amasya.

Distribution in Turkey: All over the Turkey (Önder et al., 2011).

Zoogeographical Distribution: Cyprus, Czech Republic, Egypt, Iran, Iraq, Italy, Jordan, Libya, Pakistan, Russia, Sardinia and Sicily Islands, Turkey (Nast, 1972; Önder et al., 2011).

\section{Zyginidia pullula (Boheman, 1845)}

Material examined: $4 \widehat{\jmath} 17 q+$, 28.06.2016, Çekmece Orchards, Harmanağılı Village, Suluova, Amasya.

Distribution in Turkey: Amasya, Ankara, Aydın, Bolu, Çankırı, Çorum, İzmir, İzmit, Kayseri, Manisa, Nevşehir, Sinop (Önder et al., 2011).

Zoogeographical Distribution: Europe, Iran, Kazakhstan, Moldova, Mongolia, the island of Sardinia, the North Caucasus, Turkey, Ukraine (Nast, 1972; Önder et al., 2011).

\section{Zygina flammigera (Fourcroy, 1785)}

Material examined: $1 \delta^{\Uparrow} 1 q, 27.06 .2016$, Yenimahalle, Suluova, Amasya.

Distribution in Turkey: Amasya.

Zoogeographical Distribution: Austria, Belgium, Bulgaria, Cyprus, Czech Republic, Denmark, England, Finland, France, Germany, Hungary, Ireland, Italy, Kazakhstan, Kyrgyzstan, Latvia, Moldova, Netherlands, Norway, Poland, Romania, Russia, Scotland, Slovakia, Sweden, Switzerland, Ukraine, Uzbekista (Nast, 1972).

\section{Arboridia versuta (Melichar, 1897)}

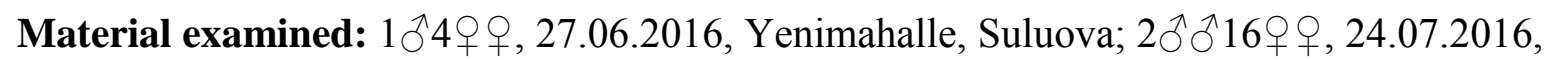

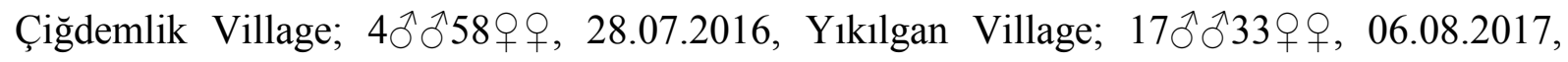

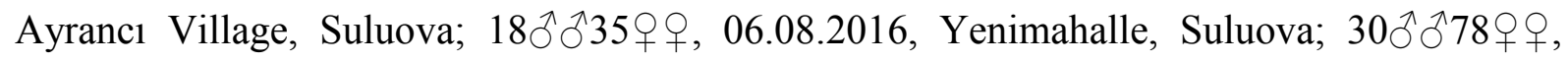
10.08.2016, Dutlupınar, Gümüşhacıköy; $9 \widehat{\jmath} 14$ 우, 18.08.2016, İpekköy; $24 \widehat{\jmath} 46$ 우,

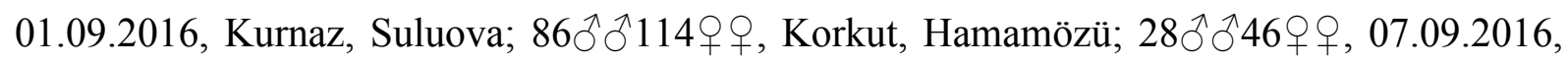
Alıcık-Merzifon, Amasya. 
Distribution in Turkey: Bitlis, Kars (Önder et al., 2011).

Zoogeographical Distribution: France, Germany, Italy, Russia, Siberia, Switzerland, Turkey, Ukraine (Önder et al., 2011).

\section{Balclutha punctata (Fabricius, 1775)}

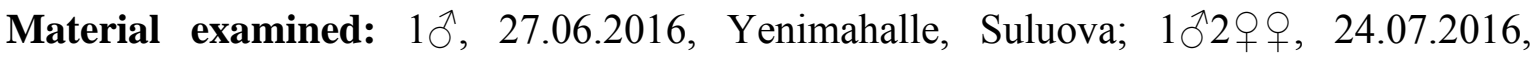

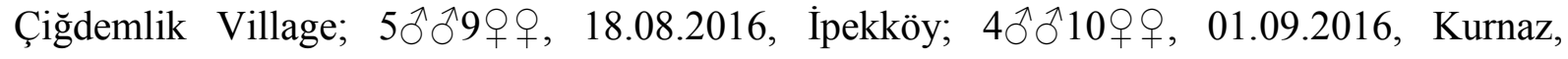

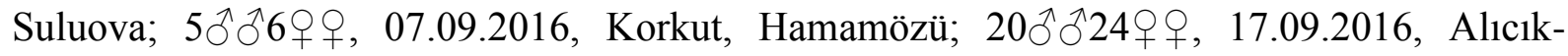
Merzifon, Amasya.

Distribution in Turkey: Adana, Adiyaman, Amasya, Ankara, Bitlis, Bolu, Diyarbakır, Erzincan, Erzurum, Hakkari, Hatay, Iğdır, Isparta, İçel, Kars, Ordu, Samsun, Sinop (Özgen and Karsavuran, 2009; Önder et al., 2011).

Zoogeographical Distribution: Afghanistan, Algeria, Austria, Azerbaijan, Bulgaria, China, Denmark, Cyprus, Czech Republic, England, Estonia, Europe, Finland, France, Germany, GreeceHungary, Iran, Ireland, Italy, Japan, Kazakhstan, Korea, Kyrgyzstan, Moldova, Mongolia, Netherlands, Norway, Poland, Portugal, Russia, Sardinia, Sicily, Slovakia, Tunisia, Turkey (Nast, 1972; Önder et al., 2011).

\section{Recilia schmidtgeni (Wagner, 1939)}

Material examined: $3 \hat{\partial} \hat{\sigma}, 18.07 .2016$, Merzifon; 1 $\hat{\sigma}, 10.08 .2016$, Dutlupınar, Gümüşhacıköy, Amasya.

Distribution in Turkey: Adana, Ankara, Diyarbakır, Erzincan, İçel, İzmir, Kırıkkale, Nevşehir, Samsun, Sinop, Şanlıurfa, Tokat (Önder et al., 2011).

Zoogeographical Distribution: Albania, Bulgaria, Crete, France, Iran, Italy, Jordan, Manchuria, Morocco, Romania, Russia, the Czech Republic, Turkey (Önder et al., 2011).

\section{Doratura homophyla (Flor, 1861)}

Material examined: $1 \lesssim 1 \uparrow$ 18.07.2016, Merzifon, Amasya.

Distribution in Turkey: Adana, Ankara, Bolu, Bursa, Diyarbakır, Eskişehir, Gaziantep, İzmir, Manisa, Van (Önder et al., 2011). 
Zoogeographical Distribution: Albania, Austria, Belgium, Bulgaria, Czech Republic, Denmark, Finland, France, Germany, Hungary, Iran, Iraq, Israel, Italy, Mongolia, Netherlands, Poland, Romania, Russia, Sweden, Turkey (Nast, 1972; Önder et al., 2011).

\section{Doratura stylata (Boheman, 1847)}

Material examined: $2 \hat{\jmath} \widehat{\jmath}, 28.07 .2016$, Y1k1lgan Village, Amasya.

Distribution in Turkey: Orta ve Doğu Anadolu, Ankara, Bayburt, Gümüşhane, Kars, Samsun, Van (Önder et al., 2011).

Zoogeographical Distribution: Algeria, England, Europe, Russia, Tunisia, Turkey (Önder et al., 2011).

\section{Platymetopius undatus (De Geer, 1773)}

Material examined: 1 , 10.08.2016, Dutlupınar, Gümüşhacıköy, Amasya.

Distribution in Turkey: Adana, Ankara, Diyarbakır, İzmit, Mardin (Önder et al., 2011).

Zoogeographical Distribution: England, Europe, Israel, Korea, Russia, Sicily, Tunisia, Turkey (Önder et al., 2011).

\section{Phlepsius intricatus (Herrich-Schäffer 1838)}

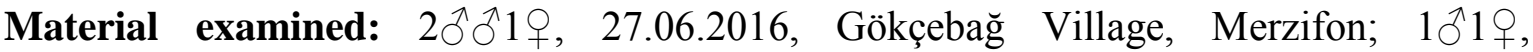

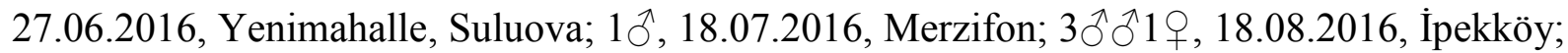
1ð̄, 01.09.2016, Kurnaz, Suluova; 1ð̂, 17.09.2016; Alıc1k-Merzifon, Amasya.

Distribution in Turkey: Amasya, Bolu, Çanakkale, Diyarbakır, Elazı̆̆ Isparta, İzmir, Kars, Kırşehir, Malatya, Mardin, Muğla, Nevşehir, Samsun (Önder et al., 2011).

Zoogeographical Distribution: Afghanistan, Albania, Austria, the Balearic Island, Bulgaria, Algeria, Czech Republic, Morocco, Palestine, France, Iraq, Iran, Spain, Italy, Cyprus, Hungary, Portugal, Romania, Russia, the island of Sardinia, Turkey, Jordan, Greece (Nast, 1972; Önder et al., 2011). 
Material examined: $1 \delta 3 q+$, 06.08.2016, Yenimahalle, Suluova; 1 $\hat{\jmath}$, 10.08.2016,

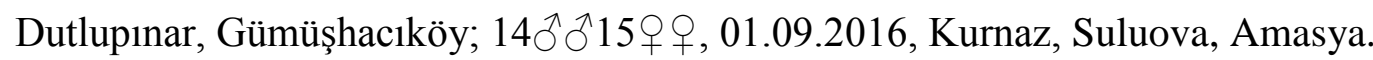

Distribution in Turkey: Amasya, Ankara, Bolu, Bursa, Denizli, Giresun, İçel, Konya, Manisa, Nevşehir, Ordu, Sakarya, Samsun, Sinop, Tokat, Trabzon (Önder et al., 2011).

Zoogeographical Distribution: Albania, Algeria, Austria, Belgium, Bulgaria, Cyprus, Czech Republic, England, France, Germany, Hungary, Ireland, Italy, Jordan, Poland, Portugal, Romania, Russia, Switzerland, Turkey (Önder et al., 2011).

\section{Euscelis lineolatus (Brullé, 1832)}

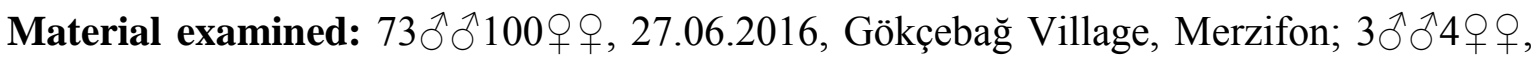
27.06.2016, Yenimahalle, Suluova; 1, 28.06.2016, Ziyaret; 30 $\widehat{\jmath} 60$ 우, 18.07.2016,

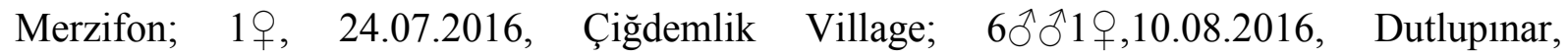
Gümüşhac1köy, Amasya.

Distribution in Turkey: Amasya, Ankara, Artvin, Balıkesir, Bursa, Denizli, İstanbul, İzmir, Kırıkkale, Kırklareli, Kırşehir, Konya, Malatya, Manisa, Niğde, Ordu, Samsun, Sinop, Tokat, Trabzon, Uşak (Zeybekoğlu, 1991; Önder et al., 2011).

Zoogeographical Distribution: Albania, Algeria, Azerbaijan, Bulgaria, France, Greece, Hungary, Iran, Ireland, Italy, Jordan, Morocco, Portugal, Sardinia, Sicily, Spain, Switzerland, the Canary Islands, the Netherlands, Tunisia, Turkey (Nast, 1972; Önder et al., 2011).

\section{Arocephalus longiceps (Kirschbaum, 1868)}

Material examined: $33 \precsim \widehat{\jmath} 28 \uparrow+$, 07.09.2016, Korkut, Hamamözü, Amasya.

Distribution in Turkey: Amasya, Ankara, Bolu, Erzincan, Erzurum, Hakkari, İzmir, Konya, Nevşehir, Samsun, Sinop (Önder et al., 2011).

Zoogeographical Distribution: Austria, Belgium, Bulgaria, Czech Republic, Denmark, France, Germany, GreeceHungary, Italy, Mongolia, Netherlands, Poland, Romania, Russia, Spain, Switzerland, Turkey (Önder et al., 2011). 


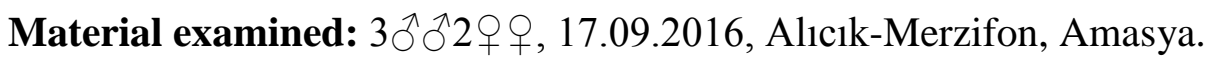

Distribution in Turkey: Adana, Ağrı, Ankara, Balıkesir, Bitlis, Edirne, Erzincan, Erzurum, Giresun, Hakkari, Isparta, Kars, Konya, Ordu, Samsun, Siirt, Sivas, Van (Önder et al., 2011).

Zoogeographical Distribution: Austria, Bulgaria, Corsica, Czech Republic, Denmark, England, Estonia, Finland, France, Germany, Greece, Holland, Hungary, Ireland, Italy, Kazakhstan, Kyrgyzstan, Norway, Poland, Romania, Russia, Slovakia, Sweden, Switzerland, Turkey, Ukraine, Uzbekistan (Nast, 1972; Önder et al., 2011).

\section{Psammotettix provincialis (Ribaut, 1925)}

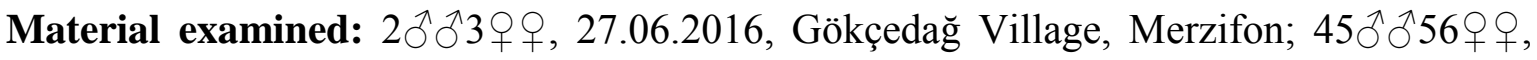

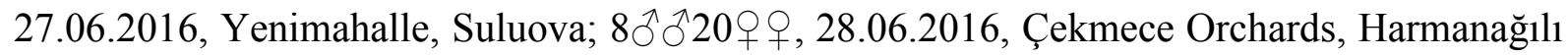

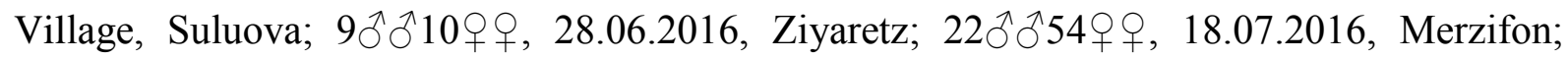

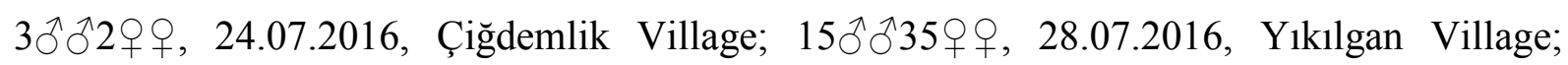

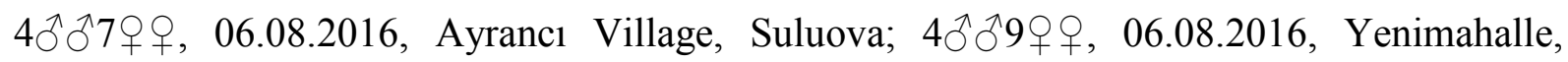

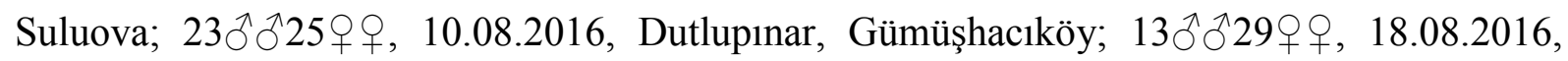
İpekköy; $4 ð ð 5$ 우, Kurnaz, Suluova, Amasya.

Distribution in Turkey: Adana, Ankara, Antalya, Aydın, Bolu, Bursa, Çankırı, Diyarbakır, Edirne, Erzurum, Hatay, İçel, İstanbul, İzmir, Kayseri, Konya, Manisa, Mardin, Nevşehir, Sakarya, Samsun, Van (Önder et al., 2011).

Zoogeographical Distribution: Afghanistan, Bulgaria, China, Cyprus, Czech Republic, France, Greece, Hungary, Iran, Italy, Mongolia, Romania, Russia, Sardinia, Sicily, Turkey (Nast, 1972; Önder et al., 2011).

\section{Artianus manderstjernii (Kirschbaum, 1868)}

Material examined: 1ठ, 27.06.2016, Gökçebağ Village, Merzifon; 1ठ, 27.06.2016 Yenimahalle, Suluova; $1 \jmath^{\Uparrow}+q$, 18.07.2016, Merzifon, Amasya.

Distribution in Turkey: Ağrı, Ankara, Balıkesir, Çankırı, Diyarbakır, Edirne, Gaziantep, İzmir, Kırklareli, Şanlıurfa,Van, Zonguldak (Önder et al., 2011). 
Zoogeographical Distribution: Bulgaria, France, Hungary, Italy, Lebanon, Romania, Russia, the Czech Republic, Turkey (Önder et al., 2011).

By this study, the species belonging to the Auchenorrhyncha which distributed in the apple orchards in Amasya were determined. The taxonomical characters of the species were similar with definitions of the related taxa in the litereture.

These are potentially pests and economically important, because they prevent the normal development of plants by absorbing the plant sap and cause the spread of plant diseases in a short time due to their dense population and the large number of species. This study is a preliminary information for agriculture. The density, damage patterns and economic importance of the species can be better demonstrated by detailed studies to be carried out in other type of agricultural fields. It is hoped that this study will contribute to future studies on Auchenorrhyncha.

\section{Acknowledgements}

This study was funded by Amasya University Scientific Research Foundation (Project No: FMB.BAP.16.0196). A part of this study was presented and printed as abstract in International Ecology Symposium 2018 (Kastamonu, Turkey) Abstract Book.

\section{References}

Asche, M. 1982. Beitrage zur Delphaciden-Fauna der Türkei (Anatolien) (Homoptera, Cicadina, Delphacidae), Marburger Entomologische Publikationen, 1(7): 71-98.

Ayaz, T., Yücel, A. 2010. Elazığ ili elma alanlarında bulunan zararlı ve yararlı arthropod türlerinin belirlenmesi üzerine araştırmalar, Harran Üniversitesi Ziraat Fakültesi Dergisi, 14(1): 9-16.

Bleicher, K., Markó, V., Orosz, A. 2006. Species composition of Cicada (Auchenorrhyncha) communities in apple and pear orchards in Hungary, Acta Phytopathologica et Entomologica Hungarica, 41(3-4): 341-355. 
Bleicher, K., Orosz, A., Cross, J., Markó, V. 2010. Survey of leafhoppers, planthoppers and froghoppers (Auchenorrhyncha) in apple orchards in South-East England, Acta Phytopathologica et Entomologica Hungarica, 45(1): 93-105.

Dlabola, J. 1957. Results of the zoological expedition of the National Museum in Prague to Turkey. 20 Homoptera, Auchenorrhyncha, Acta Entomologica Musei Nationalis Pragae, 31(469): 19-68.

Dlabola, J. 1971a. Taxonomische und chorologische Erganzungen zur Turkishchen und Iranischen Zikadenfauna (Homoptera, Auchenorrhyncha), Acta Entomologica Musei Nationalis Pragae, 14: 115-138.

Dlabola, J. 1971b. Taxonomische und chorologische Erganzungen der Zikadenfauna von Anatolien, Iran, Afganistan und Pakistan (Homoptera, Auchenorrhyncha), Acta Entomologica Bohemoslovaca, 68: 377-396.

Dlabola, J. 1974. Ubersicht der Gattungen Anoplotettix, Goldeus und Thammotettix mit Beschreibungen von 7 neuen mediterranen Arten. (Homoptera, Auchenorrhyncha), Acta Entomologica Musei Nationalis Pragae, 15: 103-130.

Dlabola, J. 1979. Neue Zikaden aus Anatolien, Iran und aus Südeuropiaeishen Laendern (Homoptera, Auchenorrhyncha), Acta Zoologica Academiae Scientiarum Hungarica, 25 (3-4): 235-257.

Dlabola, J. 1985. Zwei neue Fieberiella-Arten aus der Turkei und Spanien (Hom., Cicadellidae), Türkiye Bitki Koruma Dergisi, 9: 75-78.

Dlabola, J. 1987. Neue ostmediterrane und Iranische Zikaden taxone (Homoptera, Auchenorrhyncha), Acta Entomologica Bohemoslovaca, 84: 295-312.

Fahringer, J. 1922. Eine Rhynchoten ausbeute aus der Türkei, Kleinasien und den benachbarten Gebieten, Konowia, 1: 296-307.

Gözüaçık, C., Özgen, İ. 2018. Iğdır İli Mısır Alanlarında Bulunan Auchenorrhyncha (Homoptera, Fulgoromorpha) Türleri ve Yoğunlukları. Iğdır International Conference on Multidisciplinary Studies, 6-7 November 2018, p. 1174-1175.

Güçlü, Ş. 1996. Studies on Delphacidae (Homoptera, Auchenorrhyncha) from Turkey, Turkish Journal of Zoology, 20(4): 407-411.

Holzinger, W. E., Kammerlander, I., Nickel, H. 2003. The Auchenorrhyncha of Central Europe. Fulgoromorpha, Cicadomorpha excl. Cicadellidae. Koninklijke Brill NV, Leiden.

Kaplan, C., Tezcan, S. 2011. Cicadatra adanai Kartal, 1980 (Hemiptera: Cicadidae)'nin İzmir İli Kiraz Bahçelerinde Yayılışı, Konukçuları, Zararı ve Biyolojisi, Anadolu Doğa Bilimleri Dergisi, 2(1): 6-15. 
Kaplan, M., Yücel, A. 2014. Elazı̆̆ İli Çilek Alanlarında Belirlenen Zararlı Böcek ve Akar Türleri, Meyve Bilimi Dergisi, 1 (2): 7-14.

Karavin, M. 2012. Orta Karadeniz Bölgesi Delphacidae (Homoptera, Auchenorrhyncha) familyası türlerinin taksonomik yönden incelenmesi. $\mathrm{PhD}$ Thesis. Ondokuz Mayıs University, Institute of Science, Samsun, Turkey.

Karavin, M., Özgen, İ. 2017. Additional notes on Delphacidae, Tettigometridae and Cixidae [ Auchenorrhyncha (Insecta: Hemiptera) Fauna ] in East and Southeast Anatolia Region of Turkey, International Journal of Agriculture, Environment and Food Sciences, 1: 17-19.

Kartal, V., Zeybekoğlu, Ü. 1991. Samsun Ondokuz Mayıs University Kurupelit kampüs çevresinde Deltocephalinae (Homoptera, Auchenorrhyncha, Cicadellidae) Altfamilyası türlerinin faunistik yönden incelenmesi, Ondokuz Mayıs University Fen Dergisi, 3(1): 232257.

Kartal, V., Zeybekoğlu, Ü. 1992. Türkiye Cicadelliade (Homoptera, Auchenorryhncha) faunası için yeni türler, Doğa: Turkish Journal of Zoology, 16: 349-352.

Kartal, V., Zeybekoğlu, Ü. 1994a. Türkiye Cicadelliade (Homoptera, Auchenorryhncha) faunası için yeni tür kaydı, Turkish Journal of Zoology, 18: 37-39.

Kartal, V., Zeybekoğlu, Ü. 1994b. A new Allygidius species Alligidius nihati n. sp. (Homoptera, Auchenorryhncha, Cicadellidae, Deltocephalinae) from Turkey, Turkish Journal of Zoology, 18: 259-261.

Kartal, V., Zeybekoğlu, Ü. 1996. Türkiye'den bilinen Mocuellus dlabolai Kalkandelen, 1974 (Homoptera, Auchenorryhncha, Cicadellidae, Deltocephalinae) türü üzerine taksonomik bir araştırma, Turkish Journal of Zoology, 20: 329-331.

Kartal, V., Zeybekoğlu, Ü. 1997. Anoplotettix sahtyiancii Dlabola, 1970 (Homoptera, Auchenorryhncha, Cicadellidae, Deltocephalinae) türü üzerine taksonomik bir araştırma, Turkish Journal of Zoology, 21: 53-55.

Lodos, N., Kalkandelen, A. 1981. Preliminary list of Auchenorrhyncha with notes on distribution and importance of species in Türkei. IV. Family Issidae Spinola, Türkiye Bitki Koruma Dergisi, 5(1): 5-21.

Lodos, N., Kalkandelen, A. 1985a. Preliminary list of Auchenorrhyncha with notes on distribution and importance of species in Türkei. XVII. Family-Cicadelllidae: Deltocephalinae: Gypotini, Goniagnothini and Opsiini (Part I), Türkiye Bitki Koruma Dergisi, 9: 79-90. 
Lodos, N., Kalkandelen, A. 1985b. Preliminary list of Auchenorrhyncha with notes on distribution and importance of species in Türkei. XVIII. Family-Cicadelllidae: Deltocephalinae: Macrostelini (Part II), Türkiye Bitki Koruma Dergisi, 9(3): 146-161.

Lodos, N., Kalkandelen, A. 1985c. Preliminary list of Auchenorrhyncha with notes on distribution and importance of species in Turkey. XIX. Family-Cicadelllidae: Deltocephalinae: Deltocephalini, Scaphytopiini, Doraturini, Türkiye Bitki Koruma Dergisi, 9: 207-215.

Lodos, N., Kalkandelen, A. 1986a. Preliminary list of Auchenorrhyncha with notes on distribution and importance of species in Turkey. XX. Family-Cicadelllidae: Deltocephalinae: Fieberiellini, Stirellini and Tetartostylini, Türkiye Bitki Koruma Dergisi, 10(1): 25-32.

Lodos, N., Kalkandelen, A. 1986b. Preliminary list of Auchenorrhyncha with notes on distribution and importance of species in Turkey. XXI. Family-Cicadelllidae: Deltocephalinae: Athysanini (Part I), Türkiye Bitki Koruma Dergisi, 10(3): 131-139.

Lodos, N., Kalkandelen, A. 1986c. Preliminary list of Auchenorrhyncha with notes on distribution and importance of species in Turkey. XXII. Family-Cicadelllidae: Deltocephalinae: Athisanini (Part II), Türkiye Bitki Koruma Dergisi, 10(4): 203-211.

Lodos, N., Kalkandelen, A. 1987a. Preliminary list of Auchenorrhyncha with notes on distribution and importance of species in Turkey. XXIII. Family-Cicadelllidae: Deltocephalinae: Athisanini (Part III), Türkiye Bitki Koruma Dergisi, 11(1): 29-40.

Lodos, N., Kalkandelen, A. 1987b. Preliminary list of Auchenorrhyncha with notes on distribution and importance of species in Turkey. XXIV. Family-Cicadelllidae: Deltocephalinae: Athisanini (Part IV), Türkiye Entomoloji Dergisi, 11(2): 97-109.

Lodos, N., Kalkandelen, A. 1987c. Preliminary list of Auchenorrhyncha with notes on distribution and importance of species in Turkey. XXV. Family-Cicadelllidae: Deltocephalinae: Paralimnini (Part I), Türkiye Entomoloji Dergisi, 11(3): 151-162.

Lodos, N., Kalkandelen, A. 1987d. Preliminary list of Auchenorrhyncha with notes on distribution and importance of species in Turkey. XXVI. Family-Cicadelllidae: Deltocephalinae: Paralimnini Distant (Part II), Türkiye Entomoloji Dergisi, 11(4): 195202.

Lodos, N., Kalkandelen, A. 1988. Preliminary list of Auchenorrhyncha with notes on distribution and importance of species in Turkey. XXVII. (Addenda and Corrigenda), Türkiye Entomoloji Dergisi, 12(1): 11-22. 
Nast, J., 1972. Palaearctic Auchenorrhyncha (Homoptera). An annotated check list. Polish Scientific Publishers, Warszawa.

Ossiannilsson, F. 1978. The Auchenorrhyncha (Homoptera) of Fennoscandia and Denmark. Part 1: Introduction, infraorder Fulgoromorpha, Fauna Entomologica Scandinavica, 7(1): $1-222$.

Önder, F., Tezcan, S., Karsavuran, Y., Zeybekoğlu, Ü. 2011. Türkiye Cicadomorpha, Fulgoromorpha ve Sternorrhyncha (Insecta: Hemiptera) katoloğu, Meta Basım Matbaacılık, İzmir.

Özgen, İ., Karsavuran, Y. 2009. Diyarbakır, Elazığ ve Mardin illeri bağ alanlarında bulunan Cicadellidae (Homoptera) türleri, Türkiye Entomoloji Dergisi, 33(3): 217-240.

Özgen, İ., Topdemir, A., Mozaffarian, F. 2018. Additional notes on the some Aphrophorid Spittlebugs of Eastern Anatolia (Hemiptera: Cercopidae, Aphrophoridae), International Journal of Innovative Engineering Applications, 2(2): 60-61.

Özgen, İ., Karsavuran, Y., Zeybekoğlu, Ü., Karavin, M. 2009. Diyarbakır, Elazı̆̆ ve Mardin İlleri Bağ Alanlarındaki Auchenorrhyncha (Homoptera: Insecta) Türleri, Journal of Agricultural Faculty of Harran University, 13(3): 17- 22.

Tezcan, S., Gülperçin, N. 2010. İzmir (Kemalpaşa)'deki entegre kiraz bahçelerinde 1şık tuzaklarla yakalanan böcek faunası üzerinde bir değerlendirme, Biyoloji Bilimleri Araştırma Dergisi, 3(2): 145-147.

Tezcan, S., Gülperçin, N., Zeybekoğlu, Ü. 2010. Faunistical notes on light trap collected Auchenorrhyncha (Homoptera) species occurring in integrated cherry orchards in İzmir province of Western Turkey, Munis Entomology and Zoology, 5(2): 506-512.

Tolga, M. F., Yoldaş, Z. 2019. Hemiptera species determined in almond orchards in Mugla and Manisa provinces of Turkey and population fluctuation of Monosteira unicostata (Hemiptera:Tingidae), Akademik Ziraat Dergisi, 8(2): 209-216.

Zeybekoğlu, Ü. 1991. Orta Karadeniz Bölgesi Deltocephalinae (Homoptera, Auchenorrhyncha, Cicadellidae) Altfamilyası türlerinin taksonomik yönden incelenmesi. $\mathrm{PhD}$ Thesis. Ondokuz Mayis University, Institute of Science, Samsun, Turkey. 